\title{
The Exact Rational Solutions to a Shallow Water Wave-Like Equation by Generalized Bilinear Method
}

\author{
Minzhi Wei, Junning Cai \\ School of Information and Statistics, Guangxi University of Finance and Economics, Nanning, China \\ Email: xiaoyanxiong123@163.com
}

How to cite this paper: Wei, M. and Cai, J.N. (2017) The Exact Rational Solutions to a Shallow Water Wave-Like Equation by Generalized Bilinear Method. Journal of Applied Mathematics and Physics, 5, 715-721.

https://doi.org/10.4236/jamp.2017.53060

Received: February 17, 2017

Accepted: March 28, 2017

Published: March 31, 2017

Copyright ( 2017 by authors and Scientific Research Publishing Inc. This work is licensed under the Creative Commons Attribution International License (CC BY 4.0).

http://creativecommons.org/licenses/by/4.0/

\begin{abstract}
A Shallow Water Wave-like nonlinear differential equation is considered by using the generalized bilinear equation with the generalized bilinear derivatives $D_{3, x}$ and $D_{3, t}$, which possesses the same bilinear form as the standard shallow water wave bilinear equation. By symbolic computation, four presented classes of rational solutions contain all rational solutions to the resulting Shallow Water Wave-like equation, which generated from a search for polynomial solutions to the corresponding generalized bilinear equation.
\end{abstract}

\section{Keywords}

Rational Solution, Generalized Bilinear Equation, Shallow Water Wave Equation

\section{Introduction}

In recent years, numerous scientists committed to the research of water waves, the shallow water wave can not only describe the freedom of the shallow surface under the gravitational influence of one-way transmission, but also produce on the bottom of the deep sea. The most important of the shallow water wave in the ocean is tsunami, which generated by the huge initial disturbance, such as earthquake, leading to the large wavelength and small height ocean wave. Therefore, the wave solutions, especially the rational solutions to nonlinear differential equations have attracted more and more attentions in the worldwide. What's more, rogue wave solutions play an important role in rational solutions, which describe significant nonlinear wave phenomena in oceanography [1]. Wronskian formulation or the Casoratian formulation ([2]-[6]) usually deal with integrable equations to find their rational solutions in the literature, such as $\mathrm{KdV}$, Boussinesq, KP, Schrodinger Toda equations and Shallow water wave equ- 
ations [7]-[11]. In recent years, increasingly nonlinear differential equations are studied through the generalized bilinear equation with the generalized bilinear derivatives, for examples, KdV-like equation [12], Boussinesq-like equation [13] and KP-like equation [14].

Rational solutions to the non-integrable $(3+1)$-dimensional KP I [15] [16] and KPII [17] are also considered by different approaches. In particular, rational solutions to the $(3+1)$-dimensional KP II have been transformed into a problem of finding rational solutions to the good Boussinesq equation.

In this article, we introduce a Shallow Water Wave (SWW)-like nonlinear differential equation in terms of a generalized bilinear differential equation of Shallow Water Wave type using three generalized bilinear differential operators $D_{3, x}$ and $D_{3, t}$. We will search for polynomial solutions to the corresponding generalized bilinear equation by Maple symbolic computation and generate four classes of rational solutions to the resulting Shallow Water Wave-like equation. Four particular rational solutions will be plotted to exhibit different distributions of singularities.

\section{A SWW-Like Differential Equation}

Let us consider a generalized bilinear differential equation of SWW type:

$$
\begin{aligned}
& \left(D_{3, x} D_{3, t}-D_{3, t} D_{3, x}^{3}+D_{3, x}^{2}\right) f \cdot f \\
& =2 f_{x t} f-2 f_{x} f_{t}-6 f_{x x} f_{x t}+6 f_{x x t} f_{x}+2 f_{x x} f-2 f_{x}^{2}=0 .
\end{aligned}
$$

This is the same type bilinear equation as the SWW equation [10] [18] [19]

$$
\left(D_{x} D_{t}-D_{t} D_{x}^{3}+D_{x}^{2}\right) f \cdot f=0,
$$

with the corresponding nonlinear differential equation

$$
u_{t}-u_{x x t}-3 u u_{t}+3 u_{x} \int_{x}^{\infty} u_{t} \mathrm{~d} x+u_{x}=0 .
$$

The above differential operators are some kind of generalized bilinear differential operators introduced in [20] [21]:

$$
\begin{aligned}
& D_{p, x}^{m} D_{p, t}^{n} f \cdot f \\
= & \left.\left(\frac{\partial}{\partial x}+\alpha_{p} \frac{\partial}{\partial x^{\prime}}\right)^{m}\left(\frac{\partial}{\partial t}+\alpha_{p} \frac{\partial}{\partial t^{\prime}}\right)^{n} f(x, t) f\left(x^{\prime}, t^{\prime}\right)\right|_{x^{\prime}=x, t^{\prime}=t} \\
= & \left.\sum_{i=0}^{m} \sum_{j=0}^{n}\left(\begin{array}{c}
m \\
i
\end{array}\right)\left(\begin{array}{c}
n \\
j
\end{array}\right) \alpha_{p}^{i} \alpha_{p}^{j} \frac{\partial^{m-i}}{\partial x^{m-i}} \frac{\partial^{i}}{\partial x^{\prime(i)}} \frac{\partial^{n-j}}{\partial t^{n-j}} \frac{\partial^{j}}{\partial t^{\prime(j)}} f(x, t) f\left(x^{\prime}, t^{\prime}\right)\right|_{x^{\prime}=x, t^{\prime}=t} \\
= & \sum_{i=0}^{m} \sum_{j=0}^{n}\left(\begin{array}{c}
m \\
i
\end{array}\right)\left(\begin{array}{c}
n \\
j
\end{array}\right) \alpha_{p}^{i} \alpha_{p}^{j} \frac{\partial^{m+n-i-j} f(x, t)}{\partial x^{m-i} \partial t^{n-j}} \frac{\partial^{i+j} f(x, t)}{\partial x^{i} \partial t^{j}}, \quad m, n \geq 0 .
\end{aligned}
$$

where $\alpha_{p}^{s}$ is computed as follows:

$$
\alpha_{p}^{s}=(-1)^{r_{p}(s)}, s=r_{p}(s) \bmod p,
$$

it is necessary to point out that

$$
\alpha_{p}^{i} \alpha_{p}^{j} \neq \alpha_{p}^{i+j}, \quad i, j \geq 0 .
$$

Setting $p=3$, we have 


$$
\alpha_{3}=-1, \alpha_{3}^{2}=1, \alpha_{3}^{3}=1, \alpha_{3}^{4}=-1, \alpha_{3}^{5}=1, \alpha_{3}^{6}=1
$$

and thus

$$
\begin{aligned}
& D_{3, x} D_{3, t} f \cdot f=2 f_{x t} f-2 f_{x} f_{t}, \\
& D_{3, t} D_{3, x}^{3} f \cdot f=-6 f_{x x} f_{x t}+6 f_{x x t} f_{x}, \\
& D_{3, x}^{2} f \cdot f=2 f_{x x} f-2 f_{x}^{2} .
\end{aligned}
$$

In the case of $p=2$, which is the Hirota case, the following equations are true:

$$
\begin{aligned}
& D_{2, x} D_{2, t} f \cdot f=2 f_{x t} f-2 f_{x} f_{t}, \\
& D_{2, x}^{2} f \cdot f=2 f_{x x} f-2 f_{x}^{2}, \\
& D_{2, t} D_{2, x}^{3} f \cdot f=2 f_{x x x t} f+6 f_{x x} f_{x t}-2 f_{x x x} f_{t},
\end{aligned}
$$

which generates the standard bilinear SWW equation [18].

Motivated by the introduction on a general Bell polynomial theory [20], a dependent variable transformation is adopted:

$$
u=2(\ln f)_{x},
$$

and then can directly show that the generalized bilinear Equation (2.1) is linked to a SWW-like scalar nonlinear differential equation

$$
u_{t}+u_{x}+\frac{3}{2} u u_{x t}-\frac{3}{2} u_{x} u_{t}+\frac{1}{2} u^{2} u_{t}=0
$$

from the generalized bilinear Equation (2.1). Through the transformation (2.6), the following equality can be deduced:

$$
\frac{\left(D_{3, x} D_{3, t}-D_{3, t} D_{3, x}^{3}\right) f \cdot f}{f^{2}}=u_{t}+u_{x}+\frac{3}{2} u u_{x t}-\frac{3}{2} u_{x} u_{t}+\frac{1}{2} u^{2} u_{t},
$$

and thus, $f$ solves (2.1) if and only if $u=2(\ln f)_{x}$ presents a solution to the SWW-like Equation (2.7).

In [21] [22], transcendental functions: exponential functions and trigonometric functions have been considered to find the resonant solutions for generalized bilinear equations. In the next section, we would like to consider finding the rational solutions for SWW-like Equation (2.7), which generated from polynomial solutions.

\section{Rational Solutions}

By symbolic computation with Maple, we look for polynomial solutions, with degree of $x$ and $t$ being less than 3:

$$
f=\sum_{i=0}^{3} \sum_{j=0}^{3} c_{i, j} x^{i} x^{j}
$$

where the $c_{i, j}$ 's are constants, and present 4 classes of polynomial solutions to the generalized bilinear Equation (2.1), based on the powers of $x$, the solutions could be divided into three categories: cubic polynomials, linear polynomials and the trivial solutions. Besides the trivial solutions, those solutions, in turn, lead to four classes of rational solutions to the SWW-like (2.5) through the 
transformation (2.4). We list those classes of rational solutions as follows. The first class of rational solutions to $(2.5)$ is

$$
u_{1}=\frac{2 p}{q},
$$

where

$$
\begin{aligned}
p= & c_{10} c_{12}+c_{11} c_{12} t+c_{12}^{2} t^{2}, \\
q= & c_{10} c_{12} x+c_{11} c_{12} x t+c_{12}^{2} x t^{2}+c_{10}\left(c_{11}+c_{02}\right) \\
& +\left(c_{11} c_{02}-c_{10} c_{12}+c_{11}^{2}\right) t+c_{02} c_{12} t^{2}-c_{12}^{2} t^{3} .
\end{aligned}
$$

The second class of rational solutions to (2.5) is

$$
u_{2}=\frac{2\left(c_{11} c_{10}+c_{11}^{2} t\right)}{c_{11} c_{10} x+c_{11}^{2} x t+c_{10}\left(c_{01}+c_{10}\right)+c_{01} c_{11} t-c_{11}^{2} t^{2}},
$$

The third class of rational solutions to $(2.5)$ is

$$
u_{3}=\frac{6 p}{q},
$$

where

$$
\begin{aligned}
p= & c_{20}^{2} x^{2}+2 c_{10} c_{20} x-2 c_{20}^{2} x t+c_{10}^{2}-2 c_{20} c_{10} t+c_{20}^{2} t^{2}, \\
p= & c_{20}^{2} x^{3}+3 c_{10} c_{20} x^{2}-3 c_{20}^{2} x^{2} t+3 c_{10}^{2} x-6 c_{20} c_{10} x t+3 c_{20}^{2} x t^{2} \\
& -3\left(c_{10}^{2}-6 c_{20}^{2}\right) t+3 c_{20} c_{10} t^{2}-c_{20}^{2} t^{3}+3 c_{00} c_{10} .
\end{aligned}
$$

The fourth class of rational solutions to $(2.5)$ is

$$
u_{4}=\frac{36 p}{q},
$$

where

$$
\begin{aligned}
& p=3 c_{01} x^{2}-6 c_{01} x t+6 c_{10} x t^{2}, \\
& q=c_{01} x^{3}-3 c_{01} x^{2} t+3 c_{01} x^{2} t^{2}+18 c_{01} t-c_{01} t^{3}+18 c_{00} .
\end{aligned}
$$

Figure 1 will present the profiles of all rational solutions of (2.5) with $c_{i, j}=i^{2}+j^{2}$.

\section{Concluding Remarks}

We took a SWW-like nonlinear differential equation into consideration by the generalized bilinear equation of SWW type. Furthermore, we constructed two classes of rational solutions to the resulting SWW-like equation. A kind of generalized bilinear differential operators, which introduced in [20] [21] is the key instrument. It is an interesting work to search if there exists any Wronskian solutions and multiple type solutions to the SWW-like nonlinear Equation (2.5). At the same time, a speculation is raised, in which the four classes of rational solutions in Equations (3.2)-(3.5) would contain all rational solutions to the SWW-like nonlinear Equation (2.5), generated from polynomial solutions to the generalized bilinear Equation (2.1) with the transformation (2.4).

It is parallel to the discussion, a kind of generalized tri-linear differential equ- 


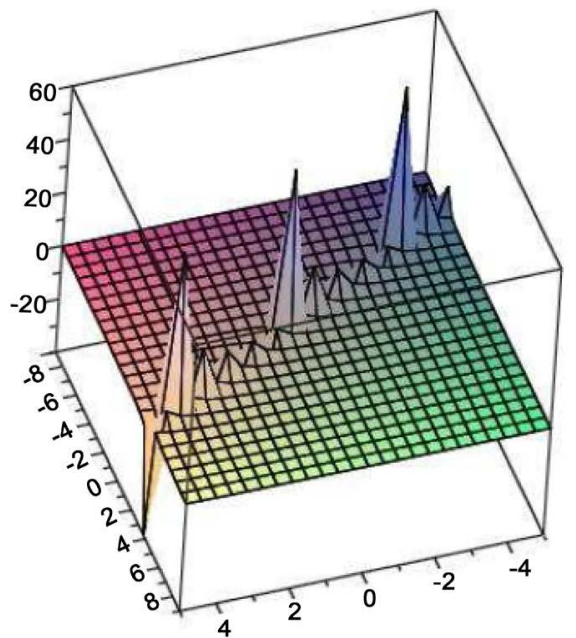

(a)

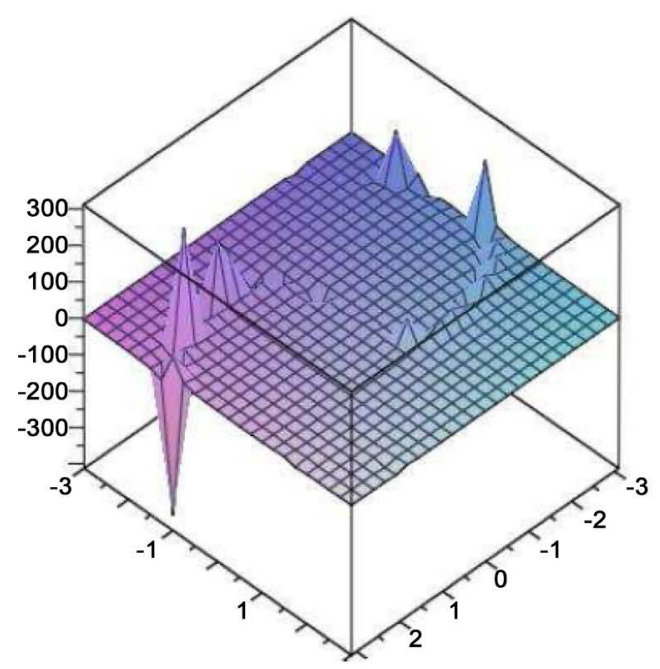

(c)

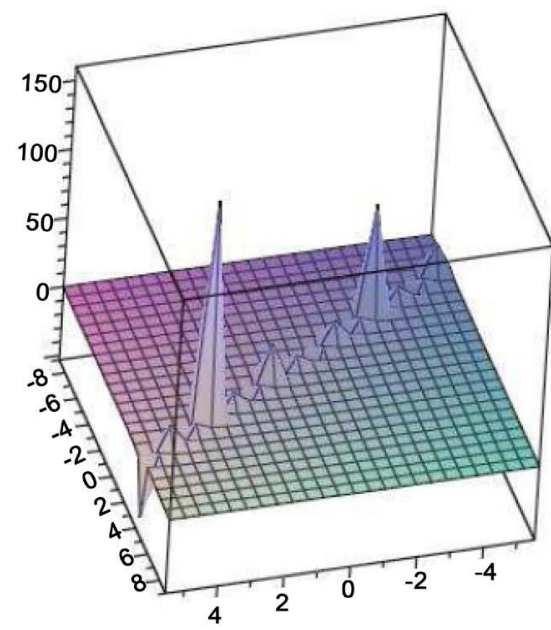

(b)

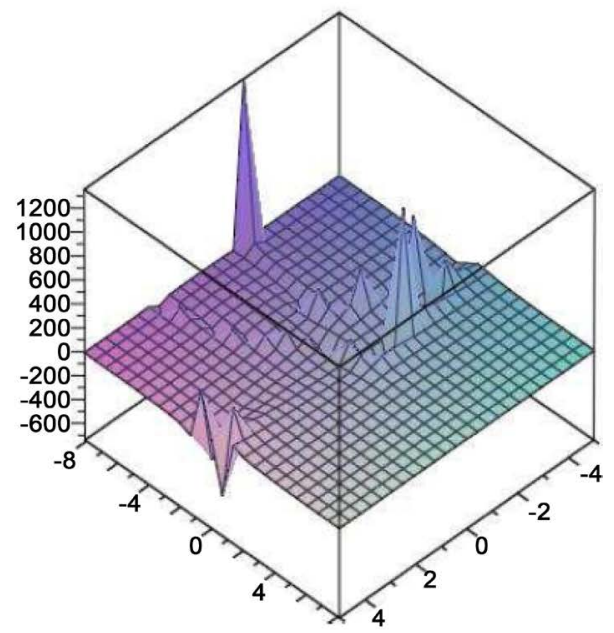

(d)

Figure 1. Pictures of the all rational solutions of (2.5) with $c_{i, j}=i^{2}+j^{2}$.

ations and their resonant solutions was considered in [23]. Their rational solutions, which include singular and non-singular wave solutions, rogue and higher-order rogue wave solutions will be a very interesting topic in generalized tri-linear differential equations in the future.

\section{Acknowledgements}

This work is supported by Guangxi College Enhancing Youths Capacity Project (KY2016LX315) and Guangxi University of Finance and Economics Youth Progresss Project (2016QNB22).

\section{References}

[1] Muller, P., Garrett, C., Osborne, A. and Waves, R. (2005) Oceanography, 18 66-75. https://doi.org/10.5670/oceanog.2005.30

[2] Ma, W.X. and You, Y. (2005) Solving the Korteweg-De Vries Equation by Its Bilinear Form: Wronskian Solutions. Transactions of the American Mathematical So- 
ciety, 357, 1753-1778. https://doi.org/10.1090/S0002-9947-04-03726-2

[3] Zhang, Y.J. (1996) Wronskian-Type Solutions for the Vector K-Constrained KP Hrerarchy. Journal of Physics A, 29, 2617-2626. https://doi.org/10.1088/0305-4470/29/10/037

[4] Hirota, R., Ohta, Y. and Satsuma, J. (1988) Wronskian Structures for Soliton Equations. Progress of Theoretical Physics Supplement, 94, 59-72. https://doi.org/10.1143/PTPS.94.59

[5] Matveev, V.B. (1992) Genernalized Wronskian Formula for Solutions of the KdV Equations: First Applications. Physics Letters A, 166, 205-208.

[6] Clarkson, P.A. and Mansfield, E.L. (1994) On a Shallow Water Wave Equation. Nonlinearity, 7, 975. https://doi.org/10.1088/0951-7715/7/3/012

[7] Nimmo, J.C. and Freeman, N.C. (1983) Rational Solutions of the Korteweg-De Vries Equation in Wronskian Form. Applied Physics Letters, 96, 443-446.

[8] Yuasa, F. (1987) Backlund Transformation of the Two-Dimensional Tada Lattice and Casorati's Determinants. Journal of the Physical Society, 56, 423-424.

[9] Hirota, R. (1986) Soliton of the Classical Boussinesq Equation and the SPH Boussinesq Equation: The Wronskian Technique. Journal of the Physical Society, 55 2137-2150. https://doi.org/10.1143/JPSJ.55.2137

[10] Chen, D.Y. (2006) Introduction to Solitons. In: Liu, Y.Q., Hu, C. and Dai, J., Eds., Journal of Applied Mathematics and Physics. Science Press, Beijing.

[11] Bagchi, B.S. and Das, A. (2010) Ganguly, New Exact Solutions of a Generalized Shallow Water Wave Equation. Physica Scripta, 82, Article ID: 025003.

https://doi.org/10.1088/0031-8949/82/02/025003

[12] Zhang, Y. and Ma, W.X. (2015) Rational Solutions to a KdV-Like Equation. Applied Mathematics and Computation, 256, 252-256. https://doi.org/10.1016/j.amc.2015.01.027

[13] Shi, C.G. and Zhao, B.Z. and Ma, W.X. (2015) Exact Rational Solutions to a Boussinesq-Like Equation in (1+1)-Dimensions. Applied Mathematics Letters, 48, 170 176.

[14] Zhang, Y.F. and Ma, W.X. (2015) A Study on Rational Solutions to a KP-Like Equation. Zeitschrift für Naturforschung A, 70, 263-268. https://doi.org/10.1515/zna-2014-0361

[15] Sinelshchikov, D.I. (2010) Comment on: New Exact Traveling Wave Solutions of the (3+1)-Dimensional Kadomtsev-Petviashvili (KP) Equation. Communications in Nonlinear Science and Numerical Simulation, 15, 3235-3236.

[16] Khalfallah, M. (2009) New Exact Traveling Wave Solutions of the (3+1) Dimensional Kadomtsev-Petviashvili (KP) Communications in Nonlinear Science and Numerical Simulation, 14, 1169-1175.

[17] Ma, W.X. (2011) Comment on the 3+1 Dimensional Kadomtsev-Petviashvili Equations. Communications in Nonlinear Science and Numerical Simulation, 16, 2663 2666.

[18] Hirota, R. (2004) The Direct Method in Soliton Theory. Cambridge University Press, Cambridge. https://doi.org/10.1017/CBO9780511543043

[19] Guo, Y.C. (2008) An Introduction to Nonlinear Partial Differential Equation, Tsinghua University Press, Beijing.

[20] Ma, W.X. (2011) Generalized Bilinear Differential Equations. Journal of Nonlinear Science, 2, 140-144.

[21] Ma, W.X. (2013) Bilinear Equations and Resonant Solutions Characterized by Bell 
Polynomials. Reports on Mathematical Physics, 72, 41-56.

[22] Zheng, H.C., Ma, W.X. and Gu, X. (2013) Hirota Bilinear Equations with Linear Subspaces of Hyperbolic and Trigonometric Function Solutions. Applied Mathematics and Computation, 220, 226-234.

[23] Ma, W.X. (2013) Trilinear Equations, Bell Polynomials, and Resonant Solutions. Frontiers of Mathematics in China, 8, 1139-1156.

https://doi.org/10.1007/s11464-013-0319-5

Submit or recommend next manuscript to SCIRP and we will provide best service for you:

Accepting pre-submission inquiries through Email, Facebook, LinkedIn, Twitter, etc. A wide selection of journals (inclusive of 9 subjects, more than 200 journals)

Providing 24-hour high-quality service

User-friendly online submission system

Fair and swift peer-review system

Efficient typesetting and proofreading procedure

Display of the result of downloads and visits, as well as the number of cited articles Maximum dissemination of your research work

Submit your manuscript at: http://papersubmission.scirp.org/

Or contact jamp@scirp.org 